\title{
Philosophiques
}

\section{L’interprétationnisme radical}

\section{Martin Montminy}

Volume 32, numéro 1, printemps 2005

Questions d'interprétation

URI : https://id.erudit.org/iderudit/011070ar

DOI : https://doi.org/10.7202/011070ar

Aller au sommaire du numéro

\section{Éditeur(s)}

Société de philosophie du Québec

\section{ISSN}

0316-2923 (imprimé)

1492-1391 (numérique)

Découvrir la revue

\section{Citer cet article}

Montminy, M. (2005). L'interprétationnisme radical. Philosophiques, 32(1), 191-206. https://doi.org/10.7202/011070ar

\section{Résumé de l'article}

J'examine la thèse défendue par Donald Davidson selon laquelle un être ne peut avoir des pensées que s'il a été en communication linguistique avec quelqu'un d'autre par le passé. Cette thèse, que j'appelle

"l'interprétationnisme radical ", dérive de la thèse $\mathbf{A}$ selon laquelle il est nécessaire d'avoir les concepts de croyance et de vérité objective pour avoir des croyances, et de la thèse $\mathbf{B}$ voulant que la communication linguistique soit requise pour l'acquisition du concept de vérité objective. En réponse à $\mathbf{A}$, je préconise un point de vue contextualiste, selon lequel les normes d'attribution de croyances dépendent du contexte conversationnel. Le contextualisme entraîne non pas que $\mathbf{A}$ est fausse, mais qu'elle doit être relative à un contexte. Je montre par ailleurs que contrairement à ce qu'affirme $\mathbf{B}$, l'interaction (linguistique) avec autrui n'est pas nécessaire pour acquérir le concept de vérité objective. Je conclus que les arguments de Davidson soutiennent au mieux l'interprétationnisme modéré, c'est-à-dire l'idée selon laquelle un être a des pensées si et seulement si ces pensées sont interprétables par un interprète pleinement informé.
Ce document est protégé par la loi sur le droit d'auteur. L'utilisation des services d’Érudit (y compris la reproduction) est assujettie à sa politique d'utilisation que vous pouvez consulter en ligne.

https://apropos.erudit.org/fr/usagers/politique-dutilisation/ 


\title{
L'interprétationnisme radical
}

\author{
MARTIN MONTMINY \\ Université d'Ottawa \\ montminy@uottawa.ca
}

\begin{abstract}
RÉSUMÉ. - J'examine la thèse défendue par Donald Davidson selon laquelle un être ne peut avoir des pensées que s'il a été en communication linguistique avec quelqu'un d'autre par le passé. Cette thèse, que j'appelle «l'interprétationnisme radical », dérive de la thèse $A$ selon laquelle il est nécessaire d'avoir les concepts de croyance et de vérité objective pour avoir des croyances, et de la thèse $\mathbf{B}$ voulant que la communication linguistique soit requise pour l'acquisition du concept de vérité objective. En réponse à $\mathbf{A}$, je préconise un point de vue contextualiste, selon lequel les normes d'attribution de croyances dépendent du contexte conversationnel. Le contextualisme entraîne non pas que $\mathbf{A}$ est fausse, mais qu'elle doit être relative à un contexte. Je montre par ailleurs que contrairement à ce qu'affirme B, l'interaction (linguistique) avec autrui n'est pas nécessaire pour acquérir le concept de vérité objective. Je conclus que les arguments de Davidson soutiennent au mieux l'interprétationnisme modéré, c'est-à-dire l'idée selon laquelle un être a des pensées si et seulement si ces pensées sont interprétables par un interprète pleinement informé.
\end{abstract}

\begin{abstract}
I examine Donald Davidson's thesis that a creature cannot have thoughts unless it has had a linguistic communication with another creature. This thesis, which I call "Radical Interpretationism", derives from the claim that (A) it is necessary to have the concepts of belief and objective truth in order to have beliefs and the claim that $(B)$ linguistic communication is required to acquire the concept of objective truth. In response to (A), I put forward a contextualist viewpoint, according to which the norms of belief attribution are context-dependent. Contextualism entails not that $(A)$ is false, but that it must be relativised to a context. I then show that contrary to what (B) says, (linguistic) interaction with others is not necessary to acquire the concept of objective truth. I conclude that Davidson's arguments at best support moderate interpretationism, that is, the view that a creature has thoughts if and only if these thoughts are interpretable by a fully-informed interpreter.
\end{abstract}

L'interprétationnisme affirme, en gros, qu'un être a des pensées (ou parle un langage) si et seulement si ces pensées (ou ce langage) sont interprétables par un interprète pleinement informé. Une version de cette thèse concerne le contenu des pensées individuelles : un être croit que $p$ si et seulement si un interprète pleinement informé jugerait que cet être croit que $p$. J'appellerai cette thèse «l'interprétationnisme modéré » et supposerai que l'interprète qu'elle mentionne est «l'un de nous» ou un être «suffisamment semblable» à nous.

Je n'essaierai pas de préciser ici cette thèse plutôt vague. L'idée de départ, difficile à caractériser de manière rigoureuse, est que les faits concernant le contenu mental (et la signification linguistique), tout comme les faits concernant la couleur par exemple, dépendent de jugements que nous produirions si certaines conditions optimales étaient réalisées; autrement dit, une explication complète de ces faits devrait faire intervenir les jugements que nous 
poserions dans ces conditions ${ }^{1}$. Bien que je trouve l'interprétationnisme modéré très plausible, je n'essayerai pas de défendre cette thèse ici. Je vais plutôt examiner une version plus radicale de l'interprétationnisme que Donald Davidson soutient depuis quelques années.

L'interprétationnisme radical affirme qu'un être ne peut avoir des pensées que s'il a été en communication linguistique avec quelqu'un d'autre par le passé. Cela veut dire qu'une personne ne peut avoir des pensées que si elle a été l'interprète du langage d'une autre personne et que son langage a lui aussi fait l'objet d'une interprétation. Le type de langage dont il est question ici doit être relativement sophistiqué et ne peut par exemple consister simplement en signaux échangés en réaction à des situations observables. Les signaux d'alarme produits par les singes vervets ${ }^{2}$ par exemple ne pourraient constituer un langage au sens où l'entend Davidson. Selon la conception qu'il propose (1999a, p. 16-17; 2001, p. 124-125), en plus de comporter des énoncés observationnels et des connecteurs logiques («et », «si..., alors... », etc.), un langage doit pouvoir exprimer une ontologie, ce qui implique, pour Davidson, la présence de quantificateurs comme «tous» et "quelques».

Malheureusement, bien qu'il ait été présenté en plusieurs endroits, l'argument de Davidson pour soutenir l'interprétationnisme radical est très succinct. Je vais examiner celui-ci en détail et signaler ses principales lacunes. Je montrerai qu'au mieux, il peut servir à étayer l'interprétationnisme modéré.

\section{L'argument}

La première partie de l'argument en faveur de l'interprétationnisme radical consiste à soutenir qu'un être ne peut avoir des pensées sans avoir des croyances. Selon Davidson, la croyance est au centre de tous les types de pensées, entendues au sens d'attitudes propositionnelles ${ }^{3}$. Le désir, le souhait, la crainte, etc., conçus comme des attitudes propositionnelles, dépendent de la croyance. Par exemple, je ne peux désirer gagner de l'argent si je ne crois pas, entre autres, que l'argent contribuera à mon bien-être; je ne peux souhaiter que la pluie cesse si je ne crois pas qu'il pleut. Cela est vrai de toute autre attitude : un être ne peut avoir une attitude sans automatiquement avoir un certain nombre de croyances qui sont directement présupposées ou impliquées par celle-ci. Mais la dépendance des attitudes par rapport aux croyances va plus loin, étant donné le holisme soutenu par Davidson. Pour qu'une attitude propositionnelle ait le contenu qu'elle a, il faut qu'elle soit liée à un réseau de croyances qui sert justement à déterminer ce contenu. Ainsi, pour craindre que le revolver soit chargé, il faut avoir plusieurs croyances à propos des revolvers : un revolver est une arme à feu, un revolver peut blesser ou tuer quelqu'un, etc. ${ }^{4}$ Par conséquent, il est nécessaire

1. Voir, entre autres, Johnston (1992) et Wright (1992) sur la notion de propriétés ou de faits qui dépendent des jugements.

2. Voir, entre autres, Cheney et Seyfarth (1990).

3. Voir Davidson (1984, p. 156-157; 1995, p. 211, p. 217-218).

4. Voir Davidson (1984, p. 156-157). 
d'avoir des croyances pour avoir des pensées, et toute condition nécessaire de la croyance est automatiquement une condition nécessaire de la pensée.

Le reste de l'argument va comme suit ${ }^{5}$. Selon Davidson, on ne peut avoir une croyance sans comprendre son contenu propositionnel. Or, comme pour Davidson, comprendre un contenu propositionnel, c'est connaître ses conditions de vérité, un être ne peut avoir de croyances que s'il comprend que celles-ci peuvent être vraies ou fausses. Cela veut donc dire que :

(A) Pour avoir des croyances, il faut posséder les concepts de croyance et de vérité objective.

Davidson soutient que l'interaction avec autrui est requise pour rendre possible l'erreur et l'acquisition du concept de vérité objective. En effet, un être qui n'a pas comparé ses réponses à l'environnement avec celles d'un autre être ne peut distinguer entre ce qui lui semble être le cas et ce qui est le cas. Selon Davidson, seule l'interaction avec autrui peut permettre une telle distinction. Or cette interaction, poursuit-il, doit être linguistique, puisque le langage est la seule façon de communiquer les contenus propositionnels d'une personne à une autre. Par conséquent :

(B) La communication linguistique est requise pour l'acquisition du concept de vérité objective ${ }^{6}$.

Je vais concentrer ma discussion sur les thèses $\mathbf{A}$ et $\mathbf{B}$. En m'appuyant sur un point de vue contextualiste selon lequel les normes d'attribution de croyances dépendent du contexte, je soutiendrai que contrairement à ce qu'affirme Davidson, A n'est valable que dans certains contextes. Je critiquerai ensuite la thèse $\mathbf{B}$ : je montrerai que l'interaction avec autrui n'est pas nécessaire pour l'acquisition du concept de vérité objective. Je soutiendrai par ailleurs que les remarques de Davidson selon lesquelles une norme de vérité n'est applicable aux pensées d'un être que si celles-ci sont comparées à celles d'un autre être impliquent, au mieux, l'interprétationnisme modéré.

\section{Les conditions nécessaires de la croyance}

Selon Davidson, les concepts de croyance et de vérité objective sont interdépendants. En effet, posséder le concept de vérité objective, c'est comprendre l'idée selon laquelle une proposition est, règle générale, vraie ou fausse indépendamment de l'attitude que l'on a à son égard. Le concept de vérité objective présuppose donc celui d'attitude propositionnelle. Mais, comme on l'a vu au début de la section précédente, le concept d'attitude propositionnelle

5. J'aurai l'occasion de clarifier les étapes cruciales de cet argument aux prochaines sections.

6. À certains endroits (1984, essai 11), la thèse de Davidson est simplement que, pour avoir des pensées, un être doit être l'interprète du langage d'un autre. Mais ailleurs (1985; 1995), Davidson soutient qu'un échange linguistique impliquant une compréhension mutuelle (adéquate) est requis. 
requiert le concept de croyance ${ }^{7}$. Cela entraîne qu'on ne peut avoir le concept de vérité objective que si l'on a le concept de croyance. Comme Davidson le souligne parfois (1984, p. 170), le concept de croyance est requis pour rendre compte de la différence entre tenir pour vrai et être vrai.

À l'inverse, on ne peut avoir le concept de croyance que si l'on a aussi le concept de vérité objective. En effet, pour posséder le concept de croyance, il est nécessaire de comprendre que la vérité ou la fausseté d'une croyance ne dépend pas simplement du fait que l'on a ou non cette croyance. En d'autres termes, le concept de croyance implique l'idée selon laquelle une croyance peut être vraie ou fausse, et que le simple fait d'avoir la croyance que $p$ n'entraîne pas que $p$. On doit donc pouvoir reconnaître qu'une croyance peut être vraie ou fausse pour avoir le concept de croyance ${ }^{8}$.

Pourquoi Davidson affirme-t-il qu'il est nécessaire de posséder le concept de croyance pour avoir des croyances? On ne peut avoir une croyance, soutient-il (1995, p. 211), si l'on ne comprend pas son contenu propositionnel. Pour Davidson, comprendre une proposition, c'est connaître ses conditions de vérité. Cela veut donc dire qu'on ne peut croire que $p$ sans connaître les conditions qui devraient être satisfaites pour que « $p$ » soit vraie. Mais pour connaître les conditions de vérité d'une proposition, il faut avoir le concept de vérité. Comme le souligne parfois Davidson, on ne peut avoir une croyance "si l'on ne comprend pas en quoi il est possible de se tromper» (1984, p. 170; trad. fr., p. $250^{9}$ ). Mais comme on vient de le voir, quiconque a le concept de vérité possède aussi le concept de croyance. On peut donc conclure qu'un être a des croyances seulement s'il possède le concept de croyance. Bref, comme on ne peut avoir une croyance sans comprendre que le contenu propositionnel de cette croyance peut être vrai ou faux, on peut conclure que :

(A) Pour avoir des croyances, il faut avoir les concepts de croyance et de vérité objective.

On a parfois l'impression que pour Davidson, A est vraie par stipulation. Davidson est en effet conscient du fait que l'on attribue souvent des croyances à des êtres (jeunes enfants, animaux, etc.) qui, semble-t-il, ne possèdent pas les concepts de croyances et de vérité objective. Cependant, soulignet-il (1995, p. 209-210), il est préférable de réserver les notions de pensées,

7. Cela n'est pas tout à fait juste, car à la section précédente il était question de la dépendance de la pensée par rapport à la croyance : le fait qu'un être ne peut avoir des pensées sans avoir des croyances n'entraîne pas, semble-t-il, qu'il ne peut posséder le concept de pensée sans posséder le concept de croyance. Mais Davidson soulignerait sans doute que pour avoir le concept de pensée, il faut comprendre la relation de dépendance entre pensées et croyances.

8. Voir, entre autres, Davidson $(1985$, p. 479-480; 1995, p. 208). Notons en passant que cette idée est tout à fait dans l'esprit du «test de la croyance fausse » (false belief task), qui suppose en général que pour avoir le concept de croyance, il faut être capable d'attribuer des croyances fausses. Voir par exemple Wimmer et Perner (1983).

9. Voir aussi Davidson (1995, p. 204, p. 208; 2001, p. 126). 
croyances, concepts, etc., aux seuls êtres qui sont conscients du fait qu'une pensée ou une croyance peut être vraie ou fausse. Pour Davidson, il n'y a donc pas de pensées du premier ordre sans pensées du deuxième ordre.

\section{Les normes d'attribution de la croyance}

Pourquoi Davidson tient-il tant à une norme d'attribution de croyances si élevée? Il se dit insatisfait du point de vue selon lequel il suffirait d'être capable de distinguer les choses qui ont une propriété donnée de celles qui ne l'ont pas pour avoir le concept désignant cette propriété. Selon un tel point de vue, fait-il valoir $(1995$, p. 209; 2001, p. 123), avoir un concept (et des croyances impliquant ce concept) se réduirait à imiter le comportement d'autrui, et même un ver de terre, par exemple, aurait les concepts de sec et d'humide. Les réserves de Davidson ne tiennent cependant pas compte du fait qu'il existe une énorme diversité d'aptitudes mentales entre celle du ver de terre ou celle du thermostat, qui peut distinguer entre "pas assez chaud » et " assez chaud ", et les nôtres. Ainsi, entre le critère de simple reconnaissance auquel satisfont le ver de terre et le thermostat et les normes davidsoniennes élevées, il existe un vaste éventail de normes possibles pour l'attribution de croyances.

Certaines fourmis (pogonomyrmex barbatus) sont capables de reconnaître la présence de congénères mortes : on a en effet observé que lorsqu'une fourmi meurt, d'autres fourmis s'emparent d'elle et la jettent dans un tas de détritus ${ }^{10}$. Nous hésiterions cependant à attribuer sur cette seule base le concept de fourmi morte à ces fourmis. Et nos doutes seraient confirmés par le fait qu'un petit bout de papier imbibé d'extraits d'acide oléique est traité de la même manière qu'un cadavre par ces fourmis. Le comportement stéréotypé des fourmis est en fait déclenché par la détection d'un seul indice.

Les animaux plus "sophistiqués " disposent toutefois de plusieurs méthodes indépendantes pour la détection d'une propriété. On peut ainsi imaginer une norme de reconnaissance «flexible ", selon laquelle la possession d'un concept (ou la possession de croyances impliquant ce concept) exige la capacité de reconnaître de plusieurs façons les choses qui ont la propriété désignée par ce concept. Selon cette norme, un être n'aurait le concept d'os, par exemple, que s'il est capable de reconnaître un os à partir de différentes modalités (vue, odorat, goût, etc.) ou dans diverses circonstances (différentes perspectives, distances, luminosités, etc.). Le critère de reconnaissance flexible consiste en fait en une famille de critères, puisque la reconnaissance dont un être fait preuve est flexible à des degrés divers. De même, la complexité des capacités mentales sous-jacentes à la reconnaissance flexible peut varier énormément. En général, toutefois, la reconnaissance flexible exige la capacité d'intégrer des indices plus ou moins variés, qui peuvent ou non provenir de différentes modalités, ainsi que la capacité de vérifier la validité d'un indice donné. On est loin du genre de capacités rudimentaires associées à la simple

10. Voir Wilson (1971, p. 278-279). 
reconnaissance observée chez les fourmis et les thermostats. La norme de reconnaissance flexible n'est bien sûr qu'une seule parmi plusieurs normes possibles pour l'attribution de croyances, situées entre la simple reconnaissance et les normes davidsoniennes.

\section{Contextualisme}

La question se pose de savoir laquelle, parmi toutes les normes possibles d'attribution de croyances, est la bonne. Je ne connais pas de principe supérieur permettant de répondre à une telle question. La locution "croire que $p$ » semble faire partie d'un groupe d'expressions comprenant, entre autres, les mots «vide» et "plat», dont les conditions d'application varient selon le contexte. Une même surface peut être correctement jugée plate ou non selon le contexte, c'est-à-dire selon les présupposés, attentes, objectifs, etc., de ceux qui portent le jugement. Par exemple, une surface asphaltée peut être convenablement plate pour un chauffeur de camion, mais jugée trop raboteuse par un adepte du patin à roues alignées. La surface en question est-elle plate ou non? Il n'existe pas de réponse adéquate indépendante du contexte. Cela ne veut évidemment pas dire que toute réponse serait acceptable : un contexte donné implique un ensemble de normes qui déterminent la mesure selon laquelle une surface plate peut comporter des inégalités.

À mon avis, il en va de même pour l'attribution de croyances : selon ce point de vue contextualiste ${ }^{11}$, l'acceptabilité d'un critère particulier pour l'attribution de croyances dépend des présupposés, attentes, objectifs, etc., de l'interprète. De même qu'il semblerait tout à fait arbitraire d'imposer un critère universel, indépendant du contexte, concernant l'application du prédicat "plat ", de même une position invariantiste, selon laquelle il existerait une seule norme valide dans tout contexte concernant l'attribution de croyances, parait arbitraire. En effet, pour défendre une norme particulière, il semble que l'on doive inévitablement s'appuyer sur nos intuitions et pratiques ordinaires d'attributions de croyances : une théorie de la croyance devrait autant que possible être conforme aux jugements que des locuteurs ordinaires poseraient concernant les croyances d'autrui ${ }^{12}$. Cela place à mon avis l'invariantiste dans une position précaire, puisque, comme le montre de façon convaincante Stich (1983), nos jugements concernant l'attribution de croyances dépendent du contexte.

Le débat stérile autour de la croyance animale illustre bien l'impasse dans laquelle se trouve l'invariantiste. La vie mentale d'un animal a plusieurs similitudes avec la nôtre : l'animal perçoit son environnement, peut faire certaines inférences, former des projets, etc. Or il semble que la meilleure explication du comportement animal invoque, entre autres, des croyances. Pourquoi

11. Stich (1983) défend un tel point de vue.

12. La conformité aux pratiques ordinaires est manifestement présupposée par les arguments philosophiques les plus influents concernant la nature des croyances. Voir, entre autres, Burge (1979), Dennett (1987), Fodor (1987), Kripke (1979) et Putnam (1975). 
Gaspard gratte-t-il frénétiquement le sol près de l'arbre? Parce qu'il veut récupérer son os et croit que celui-ci est enfoui à cet endroit. Il semble impossible d'expliquer le comportement de Gaspard, en tenant compte de sa sensibilité aux circonstances changeantes de l'environnement, sans lui attribuer des croyances. Une objection traditionnelle contre cet argument consiste à signaler l'important "fossé idéologique ${ }^{13}$ " qui sépare les animaux de nous. En effet, il n'est pas possible d'établir précisément la croyance de Gaspard, qui n'est pas en mesure de distinguer les os des artefacts qui leur ressemblent, qui ne sait rien de la fonction anatomique des os, etc. Que doit-on dire lorsque Gaspard se met à ronger un ersatz d'os ? Croit-il à tort qu'il s'agit d'un os, ou croit-il plutôt correctement qu'il s'agit d'un nonos, où "nonos " dénote l'ensemble hétérogène des os et des artefacts que Gaspard ne saurait distinguer des os ${ }^{14}$ ? L'absence de réponse déterminée à cette question est gênante pour le défenseur de la croyance animale.

L'invariantiste doit donner raison à l'un des deux camps, mais je doute fortement qu'il ait les moyens de le faire. Les intuitions invoquées par les adversaires dans ce débat ne sont pas illégitimes. Leur différend tient sans doute au fait que la notion de croyance est associée à un ensemble de caractéristiques qui ne sont pas toujours présentes simultanément. Les tenants de la croyance animale mettent l'accent sur les liens entre les croyances, les stimulations et le comportement : une croyance peut être causée par des aspects de l'environnement; une croyance (lorsque combinée à certaines autres attitudes propositionnelles) peut aussi être causalement responsable d'un comportement. Leurs opposants insistent plutôt sur les liens entre la croyance et la rationalité, la délibération, le jugement, la compréhension, etc., et soulignent que l'attribution de telles aptitudes mentales aux animaux est douteuse.

Nous sommes donc dans un état "d'indécision sémantique ${ }^{15}$ » concernant l'application du terme "croyance» aux animaux. Pour résoudre cette indécision, l'invariantiste devrait déterminer quelles sont les propriétés essentielles de la croyance. Le contextualiste évite sagement cette quête douteuse et soutient plutôt que l'indécision n'a de solution que relativement à un contexte. Comme le souligne Lewis (1994, p. 424), lorsque nous sommes

13. L'expression est de Stich (1983, p. 104-106).

14. Davidson (1984, p. 163; 1985, p. 474) écrit qu'il semble impossible d'appliquer le «test de l'intensionnalité » aux êtres dépourvus de langage : «Nous disons que le chien sait que son maître est à la maison. Mais sait-il que Monsieur Dupond (qui est son maître), ou que le président de la banque (qui est le même maître), est à la maison ? Nous ne voyons pas vraiment comment répondre, ou même comment donner un sens, à ce genre de questions » (1984, p. 163; trad. fr. p. 240). Il s'empresse cependant d'ajouter que son argument contre l'attribution de pensées aux êtres dépourvus de langage n'est pas fondé sur ce genre de considérations. Voir aussi (1985, p. 477).

15. J'emprunte cette expression à Lewis (1994, p. 424). Il existe une forme de contextualisme très populaire de nos jours en épistémologie, selon laquelle les conditions de vérité d'énoncés de la forme « $S$ sait que $p$ » varient selon le contexte de celui qui attribue la connaissance. Voir, entre autres, Cohen (1988), DeRose (1995), Lewis (1996) et Unger (1984). À mon avis, ce contextualisme est intimement lié au contextualisme de la croyance. 
dans un état d'indécision sémantique par rapport à un terme, nous sommes enclins à suivre notre interlocuteur et à consentir temporairement, pour les fins de la discussion, à son usage du terme. De même que nous pouvons accepter les normes du chauffeur de camion (ou de l'adepte du patin à roues alignées) concernant le caractère plat d'une surface, de même nous pouvons admettre les normes supposées implicitement par celui qui explique le comportement animal en invoquant des croyances.

Les aspects pragmatiques de la conversation déterminent ainsi quelles sont les caractéristiques de la croyance pertinentes à un contexte donné et contribuent par le fait même à fixer les normes d'attribution de croyances appropriées à ce contexte. Contrairement à l'invariantiste, le contextualiste n'est pas forcé de conclure que plusieurs de nos attributions ordinaires de croyances sont fausses : le fait que nous nous refusons dans certains contextes à attribuer des croyances aux animaux, par exemple lorsque nous mettons en évidence le fossé idéologique qui les séparent de nous, n'entraîne pas qu'il n'existe pas de contextes où de telles attributions sont correctes.

Doit-on conclure que la thèse $\mathrm{A}$ est fausse? Pas tout à fait. On peut suivre l'argument de Davidson et, jusqu'à "la fin de la conversation ", convenir des normes élevées qu'il propose au sujet de la croyance. Mais cela ne veut pas dire qu'un tel point de vue soit obligatoire. Autrement dit, bien qu'elle soit cohérente et plausible, la position davidsonienne sur la croyance ne règle pas la question une fois pour toute. On peut ainsi, dans d'autres contextes, admettre des normes moins élevées d'attribution de croyances et renoncer à la thèse $\mathrm{A}^{16}$.

\section{Triangulation}

La seconde thèse clé de l'argument en faveur de l'interprétationnisme radical est la suivante :

(B) La communication linguistique est requise pour l'acquisition du concept de vérité objective.

La conjonction des thèses $\mathbf{A}$ et $\mathbf{B}$ entraine qu'il faut être un interprète du langage d'autrui pour pouvoir avoir des croyances. Il importe de noter que pour Davidson, la capacité de communiquer linguistiquement avec autrui n'est pas suffisante : il faut qu'un être ait de fait eu un échange linguistique avec un autre être pour avoir des croyances. Pour défendre cette thèse quelque peu étonnante, Davidson invoque un certain type d'interaction entre deux êtres, qu'il appelle «triangulation».

16. Davidson (1985, p. 479) met en avant un argument différent en faveur de A. Il soutient que le fait d'avoir des croyances entraine la possibilité de surprise et que la surprise implique une croyance à propos de croyances, puisqu'elle requiert que l'être surpris prenne conscience du contraste entre une croyance actuelle et une croyance antérieure. Mais, contre cet argument, on pourrait objecter qu'il invoque une notion trop intellectualisée de surprise : pourquoi ne pas supposer plutôt que la surprise implique simplement une révision de croyance accompagnée d'un sursaut? 
La triangulation est une "triple interaction" impliquant deux êtres et une situation à laquelle tous deux réagissent : chacun des deux êtres observe la situation, répond à celle-ci par un certain comportement, verbal ou non, et compare sa réponse à celle donnée par l'autre être. Pour Davidson, la triangulation est accessible à des êtres non pourvus de langage et de pensées. Elle n'est donc pas une condition suffisante de la pensée. Mais elle en est une condition nécessaire, nous dit Davidson, car sans triangulation, il est impossible à un être de distinguer entre la réalité et ses propres impressions sur la réalité.

En effet, un être isolé ne peut sortir de son esprit et comparer ses réponses avec les aspects de l'environnement qui les déclenchent. Davidson nous met en garde contre un "point de vue transcendant ${ }^{17}$ ", à partir duquel il serait possible à un être isolé d'évaluer ses impressions sur le monde en les contrastant avec le monde tel qu'il est réellement : "Nous ne pouvons occuper une position extérieure à notre propre esprit; il n'y a pas de point de vue privilégié permettant de comparer nos croyances avec ce sur quoi nous supposons qu'elles portent " $(1995$, p. 208). Il n'y a donc rien, dans la situation d'un être isolé, qui permette la distinction entre une réponse adéquate et une réponse inadéquate à l'environnement. Un tel être ne peut donc posséder le concept de vérité objective ${ }^{18}$.

Lorsqu'un être interagit avec un autre, par contre, l'erreur est rendue possible, puisque les deux êtres peuvent réagir de manière différente à une même situation. La présence d'un second être qui observe le même environnement permet de comparer deux réponses qui peuvent ou non diverger. Par conséquent, seule une communauté d'au moins deux individus peut assurer la mise en place d'une norme qui permet d'appliquer le concept de vérité objective. Selon Davidson, l'interaction avec autrui est nécessaire à la fois pour «créer l'espace » permettant l'application du concept de vérité objective et pour que les participants à cette interaction acquièrent ce concept.

Mais cela n'est pas tout, nous dit Davidson. Comme je l'ai mentionné plus haut, la triangulation est une condition nécessaire mais non suffisante de la pensée. Pour établir la distinction entre ce qui est tenu pour vrai et ce qui est vrai, il faut qu'il y ait une norme publique fournie par le langage. Autrement dit, il faut que le lien qui unit les deux participants à la triangulation soit de nature linguistique. Selon Davidson, la norme de vérité doit être appliquée à des contenus propositionnels; en effet, souligne-t-il (1991,

17. L'expression est la mienne et non celle de Davidson.

18. Selon Davidson, la triangulation est aussi requise pour localiser la cause de la réponse d'un être à son environnement. En effet, souligne-t-il, s'il n'interagit pas avec autrui, il est impossible de déterminer si un être répond à un stimulus proximal ou distal (ou à une quelconque cause intermédiaire entre les deux). Je ne peux traiter adéquatement cette question dans l'espace qui m'est alloué ici. Notons tout de même que comme ma critique de $\mathbf{B}$ n'affecte pas la thèse selon laquelle la triangulation est requise pour déterminer la cause (et le contenu) des pensées, la présente discussion laisse intact un des arguments de Davidson en faveur de l'interprétationnisme radical. Voir cependant Montminy (2003), où je montre que la triangulation ne permet pas de résoudre l'indétermination entre le stimulus distal et le stimulus proximal. 
p. 201), l'erreur n'est possible que s'il y a des contenus propositionnels qui peuvent être vrais ou faux : ce sont leurs contenus propositionnels respectifs que les participants à la triangulation doivent pouvoir comparer de façon à acquérir le concept d'objectivité. Or, soutient Davidson, le langage est la seule façon de communiquer les contenus propositionnels d'une personne à une autre. Ainsi, pour qu'un être puisse comparer ses pensées à celles d'un autre, il faut qu'il se fasse l'interprète de la langue de ce dernier. Davidson n'explique pas précisément comment la norme de vérité émerge à partir de l'interaction linguistique de deux ou plusieurs êtres, mais il est clair pour lui qu'une telle norme, ainsi que la conscience de cette norme chez un individu, exigent non seulement que celui-ci ait interprété la langue d'un autre, mais aussi que sa propre langue ait été interprétée par cet autre individu ${ }^{19}$.

\section{L'application du concept d'objectivité}

Il est utile de distinguer l'argument qui concerne l'acquisition du concept d'objectivité de celui qui porte sur l'application de ce concept. La présente section est consacrée au second; j'aborderai le premier à la prochaine section. Selon Davidson, l'interaction avec autrui est nécessaire pour "créer l'espace " permettant l'application du concept de vérité objective. Son argument à cet effet est très succinct. Pour lui, seul le contraste (ou la possibilité de contraste) entre les réactions de deux êtres à un environnement commun peut donner un fondement à la distinction entre faire les choses correctement et les faire de manière incorrecte, et permettre ainsi d'appliquer une norme de vérité ${ }^{20}$.

On pourrait penser que cet argument n'a rien à voir avec la thèse $\mathbf{B}$ puisque, semble-t-il, le fait qu'un être ne puisse appliquer le concept de vérité n'entraîne pas que cet être ne possède pas le concept de vérité. En effet, pourrait-on souligner, plusieurs personnes possèdent le concept de licorne, par exemple, bien que ce concept ne puisse être appliqué (correctement) aux choses de notre monde. Le concept de vérité est toutefois particulier : $\mathrm{s}$ 'il est impossible de l'appliquer aux énoncés d'un être, on voit mal comment ceux-ci pourraient exprimer des pensées. Or un être qui n'a pas de pensées ne peut évidemment posséder le concept de vérité. Il est intéressant de noter que s'il est correct, cet argument implique l'interprétationnisme radical «directement », sans passer par les thèses intermédiaires $\mathbf{A}$ et $\mathbf{B}$. En effet, l'argument serait le suivant : puisque (i) seule l'interaction linguistique peut «créer l'espace» requis pour l'application du concept de vérité et que (ii) seul un être doté de pensées peut interagir linguistiquement avec autrui ${ }^{21}$, on peut conclure que la communication linguistique est nécessaire à la pensée, comme le veut l'interprétationnisme radical.

19. L'argument de Davidson pourrait paraître circulaire à ce point. En effet, son objectif est d'énoncer les conditions qui rendent possible la pensée; or ces conditions (plus particulièrement, la communication linguistique) exigent elles-mêmes l'existence de contenus propositionnels. Mais pour Davidson, le langage et la pensée émergent simultanément.

20. Voir Davidson (1999a, p. 13; 1999b, p. 731; 1999c, p. 208).

21. Cette seconde prémisse va de soi selon Davidson (1984, p. 55). 
Malheureusement, ce que cet argument montre au mieux, c'est qu'une comparaison entre deux systèmes de croyances doit pouvoir être effectuée pour que la norme de vérité puisse être appliquée, et non pas qu'une comparaison doit avoir été effectuée. Autrement dit, l'argument implique au mieux l'interprétationnisme modéré et non l'interprétationnisme radical. L'analogie avec les concepts de couleur est utile : bien que le caractère rouge d'une surface dépende essentiellement du jugement d'un observateur normal, cela n'entraîne pas qu'une surface doive avoir été vue par un tel observateur pour qu'elle soit rouge. Pourquoi en serait-il autrement du concept de vérité objective? Les brèves remarques de Davidson ne montrent pas qu'un échange linguistique doit avoir eu lieu pour que ce concept soit applicable.

La position de Davidson comporte en outre une importante difficulté. Ce dernier ne voudrait certainement pas dire que pour qu'un être ait telle ou telle croyance, il faut que cette croyance particulière ait été attribuée par un interprète. En effet, il ne nierait pas que nous pouvons avoir des croyances que nous n'avons jamais communiquées à quiconque. Sa position est donc que pour qu'un être ait des croyances, il faut qu'un certain sous-ensemble de celles-ci aient été communiquées à autrui. Le sous-ensemble en question peut évidemment varier d'un individu à l'autre. La norme de vérité est applicable aux croyances non communiquées d'un être, pourvu que celui-ci ait communiqué d'autres croyances à autrui. Cela veut dire que l'échange linguistique contribue à conférer le statut de croyances non seulement aux états mentaux communiqués, mais aussi à ceux qui ne le sont pas. Il s'agit là d'un point de vue plutôt bizarre, puisque l'échange linguistique pourrait avoir porté sur des sujets qui n'ont rien à voir avec les croyances non communiquées. Je vois mal comment les bénéfices tirés d'un échange linguistique peuvent avoir une telle répercussion sur un nombre indéfiniment grand de croyances (non communiquées). L'interprétationnisme modéré évite cette conséquence contraire à l'intuition, puisqu'il affirme qu'un être a telles et telles croyances (non communiquées) si et seulement si un interprète pleinement informé jugerait que cet être a ces croyances. Les croyances en question n'ont donc pas à attendre qu'un échange linguistique ait eu lieu avec un interprète pour exister (en tant que croyance).

\section{L'acquisition du concept d'objectivité}

L'autre argument avancé par Davidson pour soutenir la thèse $\mathbf{B}$ concerne l'acquisition du concept d'objectivité : seul l'échange linguistique permet d'acquérir ce concept, puisque deux interlocuteurs peuvent réagir de façon différente à leur environnement et en venir ainsi à comprendre que leurs propres réactions peuvent être erronées. Cependant, comme l'ont signalé un certain nombre d'auteurs, il semble que les moyens dont dispose un être isolé pour acquérir le concept de vérité objective sont tout aussi efficaces que ceux qui s'offrent aux participants à la triangulation. J'en vois au moins trois. 
Tout d'abord, l'être isolé peut comparer sa réaction présente à une réaction passée 22 . Robinson observe un bâton à moitié plongé dans l'eau. Son impression est que le bâton est courbé. Il observe ensuite le même bâton d'un angle différent. Le bâton lui paraît maintenant être droit. Il semble que ses deux observations peuvent permettre à Robinson de constater une différence entre ce qui lui semble être le cas et ce qui est le cas, puisque au moins une de ses deux impressions doit être erronée.

Un deuxième procédé accessible à l'être isolé consiste à contraster sa réaction présente avec une généralisation qu'il a jusqu'ici admise. Comme à chaque fois qu'il jette une pierre dans l'étang, celle-ci coule à pic, Robinson s'attend à ce que la pierre qu'il s'apprête à lancer dans l'étang coule à pic elle aussi. Mais aujourd'hui l'étang est gelé et, à la surprise de Robinson, la pierre rebondit sur la surface. L'observation faite par Robinson va à l'encontre de la généralisation selon laquelle toute pierre jetée dans l'étang coule à pic et peut, semble-t-il, lui permettre de distinguer entre ses attentes et ce qui se passe réellement.

Finalement, l'être isolé peut comparer sa réaction présente à celle qu'il (ou un autre être) pourrait avoir s'il observait la même situation selon une perspective différente. Il peut en effet imaginer comment les choses lui sembleraient être si sa distance ou son angle d'observation était différent, si l'éclairage n'était pas le même, etc. Le cheval que Robinson aperçoit au loin semble avoir trois pattes. Robinson peut imaginer comment ce cheval lui apparaîtrait si son point de vue était différent : peut-être qu'il lui semblerait plutôt que l'animal a quatre pattes. Une telle «expérience de pensée » peut, apparemment, permettre à Robinson de prendre conscience du fait qu'il peut y avoir un écart entre la façon dont les choses sont et la façon dont elles lui apparaissent.

Un contraste entre son impression présente et une impression passée, une généralisation ou une impression possible ne peut bien sûr garantir l'acquisition du concept d'objectivité. L'être isolé pourrait ne pas relever le conflit ou l'apparence de conflit et admettre une forme de subjectivisme, selon laquelle la réalité est toujours telle qu'elle lui apparaît être. Il pourrait par exemple se dire que puisque son impression passée était que le bâton était courbé, le bâton était alors courbé, et que le bâton est maintenant droit, étant donné que son impression présente est que le bâton est droit ${ }^{23}$. Les trois méthodes que je viens de décrire semblent donc vulnérables à l'objection de Davidson présentée à la section 5 : comme le sujet isolé ne peut occuper une position extérieure à lui-même lui permettant de comparer ses impressions à la réalité, il ne peut rejeter le point de vue subjectiviste voulant que la réalité soit fixée par les impressions qu'il en a. Toute tentative d'attribuer à l'être isolé un concept d'objectivité serait vouée à l'échec, puisque ce dernier ne serait jamais en mesure d'aller au-delà de ses impressions sur le monde.

22. Voir Heil (1992, p. 217) et Child (1994, p. 19) pour une suggestion de ce type.

23. De façon similaire, il pourrait se dire que si son impression était que le bâton est courbé, alors le bâton serait courbé. 
Cette objection ne me paraît pas convaincante. Notons d'abord que le participant à la triangulation n'y échapperait pas. En effet, contrairement à ce que Davidson laisse parfois entendre, ce dernier n'est pas en mesure de comparer ses impressions sur le monde avec celles de l'autre participant. Pour ce faire, il faudrait qu'il soit capable d'occuper une position extérieure à luimême : au mieux, il peut comparer ses impressions sur le monde avec ses impressions sur les impressions de l'autre participant. Je ne vois donc pas en quoi l'interaction avec autrui permet à un individu de se tirer de l'impasse dans laquelle se trouve l'être isolé : aucun ne peut contraster ses impressions sur le monde avec le monde tel qu'il est réellement. Les raisons invoquées par Davidson pour nier qu'il soit possible à l'être isolé d'acquérir le concept d'objectivité s'appliquent donc tout autant aux participants à la triangulation.

Mais laissons cette difficulté et supposons que les participants à la triangulation puissent réellement comparer leurs impressions respectives. Cela ne leur permet pas d'éviter une variante de l'objection présentée plus haut : la triangulation ne fait que remplacer le spectre du subjectivisme par celui du relativisme. Supposons en effet que Robinson ait l'impression que le bâton est courbé et que Vendredi ait l'impression qu'il est droit. Robinson pourrait se dire que puisque Vendredi a l'impression que le bâton est courbé, du point de vue de Vendredi, le bâton est courbé, alors que de son point de vue à lui, il ne l'est pas, car son impression est que le bâton est droit. De même que les impressions de l'être isolé ne lui permettent pas d'exclure le subjectivisme, de même les impressions des participants à la triangulation ne leur permettent pas de rejeter le relativisme.

Manifestement, il y a quelque chose qui cloche : l'objection que je viens de présenter semble entraîner que personne ne peut posséder le concept d'objectivité, puisque personne n'est en mesure de réfuter le subjectivisme (ou le relativisme), sur la base de ses impressions sur la réalité. Mais il importe de distinguer deux tâches qui, dans les écrits de Davidson, ne sont pas nettement différenciées. La première consiste à établir la norme de vérité (pour nos croyances et énoncés) : c'est une tâche que ni l'être isolé ni les participants à la triangulation ne peuvent accomplir de façon parfaitement satisfaisante (ou, si l'on veut, avec le genre de certitude qui permettrait de réfuter le subjectivisme ou le relativisme), puisque pour ce faire, ils devraient avoir accès à un point de vue transcendant leur permettant de comparer leurs croyances avec ce sur quoi elles portent. La seconde tâche est beaucoup moins exigeante : elle consiste simplement à comprendre que nos croyances peuvent être erronées. L'erreur dans l'objection présentée plus haut est de trop demander pour la possession du concept d'objectivité : ce concept exige non pas de pouvoir démontrer que la réalité n'est pas entièrement modelée sur nos impressions ou jugements, mais de comprendre que ces impressions ou jugements peuvent être erronés. Or l'une ou l'autre des trois méthodes présentées plus haut peut permettre à l'être isolé de comprendre cette possibilité. Par conséquent, le fait qu'il ne peut occuper un point de vue transcendant n'empêche pas l'être isolé d'acquérir le concept d'objectivité. 


\section{Conclusion}

J'ai examiné l'interprétationnisme radical que défend Davidson depuis quelques années. Plus particulièrement, j'ai remis en question deux prémisses cruciales de cette doctrine, à savoir la thèse A selon laquelle il est nécessaire d'avoir les concepts de croyance et de vérité objective pour avoir des croyances et la thèse $B$ qui affirme que la communication linguistique est requise pour l'acquisition du concept de vérité objective. À la place de $\mathrm{A}$, je préconise un point de vue contextualiste, selon lequel les normes d'attribution de croyances varient selon le contexte. Mon contextualisme me permet de concéder A à Davidson, sans toutefois admettre que A soit valable dans tous les contextes. Autrement dit, selon le contextualisme, une fois qu'elles ont été mises en rapport avec un contexte, les normes d'attribution de croyances davidsoniennes sont tout à fait compatibles avec le fait que, dans des contextes ordinaires, il peut être correct d'attribuer des croyances aux êtres qui ne possèdent pas les concepts de croyance et de vérité objective.

J'ai par ailleurs montré que l'interaction avec autrui n'est pas nécessaire pour comprendre que nos propres impressions sur la réalité peuvent être erronées. Il va de soi que la comparaison entre nos réponses et celles d'une autre personne peut faciliter une telle compréhension. Un être isolé dispose toutefois d'au moins trois méthodes différentes lui permettant de constater que les réactions qu'il a par rapport à son environnement ne sont pas nécessairement correctes. Ces méthodes ne lui permettent pas de démontrer qu'il y a une réalité indépendante de son esprit et quelle est la nature précise de cette réalité, mais une telle démonstration n'est pas requise pour la possession du concept de vérité objective.

Comment la norme de vérité ou, si l'on veut, les conditions d'application du concept de vérité objective, sont-elles fixées? Cette question dépasse le cadre de mon exposé. Je peux tout de même signaler que l'interprétationnisme modéré me semble une avenue prometteuse ${ }^{24}$. Selon ce point de vue, la question du contenu des croyances d'un être dépend essentiellement de l'interprétation que nous en ferions. Ainsi, l'application d'une norme de vérité aux impressions et jugements d'un être n'aurait de sens que dans le cadre d'une comparaison entre ses pensées et les nôtres. Mais l'interprétation dont il est question ici en est une qui pourrait avoir lieu et non pas une interprétation qui, de fait, a eu lieu ${ }^{25}$.

24. Je développe cette idée plus à fond dans Montminy (à paraître).

25. Je tiens à remercier Sherri Irvin, Maurice Lagueux, Daniel Laurier, Michel Seymour, ainsi que les deux lecteurs anonymes de cette revue pour leurs commentaires utiles sur ce texte. Je remercie aussi le CRSH de m'avoir accordé une subvention de recherche. 


\section{Bibliographie}

Burge, T. «Individualism and the Mental », dans A. P. French, T. Uehling et H. Wettstein, dir., Midwest Studies in Philosophy, 4, Minneapolis, University of Minnesota Press, 1979, p. 73-121.

Cheney, D. L. et R. M. Seyfarth, How Monkeys See the World: Inside the Mind of Another Species, Chicago, University of Chicago Press, 1990. Child, W. Causality, Interpretation and the Mind, Oxford, Clarendon Press, 1994.

Cohen, S. "How to be a Fallibilist ", Philosophical Perspectives, 2, 1988, p. 581-605.

Davidson, D. Inquiries into Truth and Interpretation, Oxford, Clarendon Press, 1984. (Trad. fr. Enquêtes sur la vérité et l'interprétation, Nîmes, J. Chambon, 1993.)

. "Rational Animals", dans E. Lepore et B. McLaughlin, dir., Actions and Events : Perspectives on the Philosophy of Donald Davidson, Oxford, Basil Blackwell, 1985, p. 473-481. (Trad. fr. "Animaux rationnels " dans Paradoxes de l'irrationalité, Combas, Éditions de l'Éclat, p. 63-75.)

. "Epistemology Externalized», Dialectica, 45, 1991, p. 191-202.

. "The Problem of Objectivity", Tijdschrift voor Filosofie, 57, 1995, p. 203-220.

. "The Emergence of Thought", Erkenntnis, 51, 1999a, p. 7-17.

. "Reply to Dagfinn Føllesdal ", dans L.E. Hahn, dir., The Philosophy of Donald Davidson, Chicago et La Salle, Open Court, 1999b, p. 729-732. . "Reply to Thomas Nagel ", dans L.E. Hahn, dir., The Philosophy of Donald Davidson, Chicago et La Salle, Open Court, 1999c, p. 207-209. . "What Thought Requires", dans J. Branquinho, dir., The Foundations of Cognitive Science, Oxford, Clarendon Press, 2001, p. 121-132.

Dennett, D. C. The Intentional Stance, Cambridge (Mass.), MIT Press, 1987.

(Trad. fr. La stratégie de l'interprète, Paris, Gallimard, 1990.)

DeRose, K. "Solving the Skeptical Problem », Philosophical Review, 104, 1995, p. 1-52.

Fodor, J. A. Psychosemantics, Cambridge (Mass.), MIT Press, 1987.

Heil, J. The Nature of True Minds, Cambridge, Cambridge University Press, 1992.

Johnston, M. «Objectivity Refigured : Pragmatism Without Verificationism », dans J. Haldane et C. Wright, dir., Reality, Representation and Projection, Oxford, Oxford University Press, 1992, p. 85-130.

Kripke, S. "A Puzzle about Belief ", dans A. Margalit, dir., Meaning and Use, Dordrecht, Reidel, 1979, p. 239-283.

Lewis, D. "Reduction of Mind ", dans S. Guttenplan, dir., A Companion to the Philosophy of Mind, Oxford, Basil Blackwell, 1994, p. 412-431.

- . "Elusive Knowledge ", Australasian Journal of Philosophy, 74, 1996, p. 549-567. 
Montminy, M. "Triangulation, Objectivity and the Ambiguity Problem ", Crítica, 35, 2003, p. 25-48.

. "Meaning Skepticism and Normativity ", Journal of Philosophical Research, à paraître.

Putnam, H. "The Meaning of "Meaning" ", dans Mind, Language and Reality, Cambridge, Cambridge University Press, 1975, p. 215-271.

Stich, S. From Folk Psychology to Cognitive Science, Cambridge (Mass.), MIT Press, 1983.

Unger, P. Philosophical Relativity, Minneapolis, University of Minnesota Press, 1984.

Wilson, E. O. The Insect Societies, Cambridge (Mass.), Harvard University Press, 1971.

Wimmer, H. et J. Perner. "Beliefs about Beliefs : Representation and Constraining Function of Wrong Beliefs in Young Children's Understanding of Deception ", Cognition, 13, 1983, p. 103-128.

Wright, C. Truth and Objectivity, Cambridge (Mass.), Harvard University Press, 1992. 